\title{
ON REGULAR GROUP RINGS
}

\section{MAURICE AUSLANDER ${ }^{1}$}

Let $G$ be a multiplicative group, $K$ a commutative ring with unit, and $K(G)$ the group ring of $G$ with respect to $K$. We say that $K(G)$ is regular if given an $x$ in $K(G)$, there is a $y$ in $K(G)$ such that $x y x=x$. Using a homological characterization of regular rings which was found independently by M. Harada [2, Theorem 5] and the author, we prove that if $G$ is locally finite, then $K(G)$ is regular if and only if $K$ is regular and is uniquely divisible by the order of each element in $G$. More generally we show that if $K(G)$ is regular, then $G$ is a torsion group and $K$ is a regular ring which is uniquely divisible by the order of each element in $G$. A nonhomological proof of these results has been given by J. McLaughlin (unpublished). In conclusion, we show that if $G$ is a commutative group and $K$ is a field of characteristic not dividing the order of any element in $G$, then the weak global dimension of $K(G)$ equals the rank of $G$. For the most part we follow the conventions and notations in [1].

Let $R$ be a ring (with unit) and $A$ a left $R$-module. The weak left dimension of $A$ is defined as follows (see [1, Chapter VI, Exercise 3]):

$$
-1 \leqq \text { w. } 1 . \operatorname{dim}_{R} A \leqq \infty
$$

where w. $1 . \operatorname{dim}_{R} A<n$ if and only if $\operatorname{Tor}_{n}^{R}(C, A)=0$ for all right $R$ modules $C$. Since the $\operatorname{Tor}_{n}^{R}(C, A)$ are half exact functors of $C$ which commute with direct limits, it follows from [1, V, Exer. 9] that we may restrict $C$ to be of the form $R / I$ where $I$ is a right ideal in $R$. For a right $R$-module $C$, we define w. r. $\operatorname{dim}_{R} C$ similarly.

The weak global dimension of $R$ is defined as follows:

$$
0 \leqq \mathrm{w} \cdot \operatorname{gl} \cdot \operatorname{dim} R \leqq \infty
$$

where w. gl. $\operatorname{dim} R<n$ if and only if $\operatorname{Tor}_{n}^{R}=0$. We have

$$
\text { w. gl. } \begin{aligned}
\operatorname{dim} R & =\sup _{A} \text { w. l. } \operatorname{dim}_{R} A=\sup _{C} \text { w. r. } \operatorname{dim}_{R} C \\
& =\sup _{I} \text { w. } 1 . \operatorname{dim} R / I=\sup _{J} \text { w. r. } \operatorname{dim} R / J,
\end{aligned}
$$

where $A$ and $C$ range over all left and right $R$-modules respectively, and $I$ and $J$ range over all left and right ideals respectively. It follows therefore that the w. $1 . \operatorname{dim} R<n$ if and only if $\operatorname{Tor}_{n}^{R}(R / J, R / I)=0$ for all left ideals $I$ and right ideals $J$.

Received by the editors July 12, 1956 .

1 Part of the work in this paper was done while the author was on a National Science Foundation Fellowship. 
THEOREM 1. For a ring $R$ (with unit), the following statements are equivalent:

(a) $R$ is regular,

(b) For every left ideal $I$ and right ideal $J$ in $R$, we have $J I=J I$,

(c) w. gl. $\operatorname{dim} R=0$.

Proof. (a) $\Rightarrow(\mathrm{b})$. It is clear that $J \cap I \supset J I$. Let $x$ be in $J \cap I$. Then there is a $y$ in $R$ such that $x y x=x$. Since $x y$ is in $J$ and $x$ as in $I$, we have that $x=x y x$ is in $J I$.

(b) $\Rightarrow(\mathrm{a})$. Let $x$ be in $R$. Since $x$ is in $x R \cap R x=x R x$, there is a $y$ in $R$ such that $x y x=x$.

(b) $\Leftrightarrow$ (c). By [1, Chapter VI, Exercise 19] we know that

$$
\operatorname{Tor}_{I}^{R}(R / J, R / I)=J \cap I / J I .
$$

Therefore the w. gl. $\operatorname{dim} R=0$ if and only if $J \cap I=J I$.

Proposition 2. Let $R$ be a subring of the ring S. Suppose w. $r . \operatorname{dim}_{R} S$ $=0$. Then for all left $R$-modules $A$ and all right $S$-modules $C$ we have

w. $1 . \operatorname{dim}_{R} A \geqq$ w. $1 . \operatorname{dim}_{S} S \otimes_{R} A$,

w. r. $\operatorname{dim}_{R} C \leqq$ w. r. $\operatorname{dim}_{S} C$.

Further, if $S$ considered as a two-sided $R$-module contains $R$ as a two-sided direct summand, then for all left $R$-modules $A$

$$
\text { w. } 1 . \operatorname{dim}_{R} A=\text { w. } 1 . \operatorname{dim}_{S} S \otimes_{R} A
$$

and thus

$$
\text { w. gl. } \operatorname{dim} S \geqq \text { w. gl. } \operatorname{dim} R \text {. }
$$

Proof. Since w. r. $\operatorname{dim}_{R} S=0$, we have by $[1$, Ch. VI, 4.1.1] that

$$
\operatorname{Tor}_{n}^{R}(C, A) \approx \operatorname{Tor}_{n}^{S}\left(C, S \otimes_{R} A\right)
$$

for all $n \geqq 0$. The first two inequalities follow immediately from this isomorphism.

Suppose $D$ is a right $R$-module. Then $C=D \otimes_{R} S$ is a right $S$-module. Since $S$ as a two-sided $R$-module contains $R$ as a two-sided $R$ module, we have that $D$ is isomorphic to a direct summand of $C$ when $C$ is considered as a right $R$-module. Since $\operatorname{Tor}_{n}^{R}$ commutes with direct sums we have that $\operatorname{Tor}_{n}^{R}(D, A) \neq 0$ implies that $\operatorname{Tor}_{n}^{S}(C, A)$ $\neq 0$. Therefore, we have w. $1 . \operatorname{dim}_{R} A=$ w. $1 . \operatorname{dim}_{S} S \otimes_{R} A$. The rest of the proposition follows from the definition of weak global dimension.

Proposition 3. Let $G$ be a group, $G^{\prime}$ a subgroup of $G$, and $K$ a com- 
mutative ring. Then we have

(a) $K(G)$ is a free left $K\left(G^{\prime}\right)$-module and thus

$$
\text { 1. } \operatorname{dim}_{K\left(G^{\prime}\right)} K(G)=0=\text { w. } 1 . \operatorname{dim}_{K\left(G^{\prime}\right)} K(G) \text {. }
$$

(b) $K(G)$ is a free right $K\left(G^{\prime}\right)$-module and thus

$$
\text { r. } \operatorname{dim}_{K\left(G^{\prime}\right)} K(G)=0=\text { w. r. } \operatorname{dim}_{K\left(G^{\prime}\right)} K(G) \text {. }
$$

(c) $K(G)$, considered as a two-sided $K\left(G^{\prime}\right)$-module, contains $K\left(G^{\prime}\right)$ as a two-sided direct summand.

Thus if we set $R=K\left(G^{\prime}\right)$ and $S=K(G)$, the results of Proposition 2 remain valid.

Proof. (a) It is easily seen that any system of representatives for the left cosets of $G^{\prime}$ in $G$, is a basis for $K(G)$ considered as a left $K\left(G^{\prime}\right)$-module.

(b) Analogous argument to that used in (a).

(c) We may assume that $F=G-G^{\prime}$ is not empty. Since $G^{\prime} F=F G^{\prime}$ $=F$, the $K$-submodule generated by $F$ in $K(G)$, which we will denote by $K(F)$, is a two-sided $K\left(G^{\prime}\right)$-submodule of $K(G)$. It is clear that $K(F) \cap K\left(G^{\prime}\right)=0$. Thus $K\left(G^{\prime}\right)$ is a two-sided direct summand of $K(G)$.

We define the ring epimorphism $\epsilon: K(G) \rightarrow K$ by $\epsilon\left(\sum k_{i} g_{i}\right)=\sum k_{i}$. This homomorphism is called the unit augmentation. From now on we consider $K$ as a two-sided $K(G)$-module as follows: $x k=\epsilon(x) k$ and $k x=k \epsilon(x)$ for all $k$ in $K$ and $x$ in $K(G)$.

Proposition 4. Let $K$ be a left $K(G)$-module. Then we have

w. gl. $\operatorname{dim} K \leqq$ w. gl. $\operatorname{dim} K(G) \leqq$ w. $1 \cdot \operatorname{dim}_{K(G)} K+$ w. gl. $\operatorname{dim} K$.

Proof. The first inequality follows from Proposition 3 by setting $G^{\prime}=\{1\}$. Thus in proving the second inequality, we need only consider the case where both w. $\operatorname{dim}_{K(G)} K=r$ and w. gl. $\operatorname{dim} K=s$ are finite. Suppose $A$ and $B$ are right and left $K(G)$-modules respectively. Let

$$
0 \rightarrow X_{s} \rightarrow X_{s-1} \rightarrow \cdots \rightarrow X_{0} \rightarrow A \rightarrow 0
$$

be an exact sequence of right $K(G)$-modules where the $X_{i}$ are $K(G)$ projective for $i=0, \cdots, s-1$. Then we have by [1, Chapter $\mathrm{V}, 7.2]$ that

$$
\operatorname{Tor}_{p+s}^{K(G)}(A, B) \approx \operatorname{Tor}_{p}^{K(G)}\left(X_{s}, B\right)
$$

for all $p>0$.

Since $K(G)$ is a projective $K$-module, each of the $X_{i}, i=1, \cdots, s-1$, 
is a projective module when considered as $K$-modules. Applying [ 1 , Chapter V, 7.2] again we have

$$
\operatorname{Tor}_{p+s}^{K}(A, B) \approx \operatorname{Tor}_{p}^{K}\left(X_{s}, B\right)
$$

for all $p>0$. Since w. gl. $\operatorname{dim} K=s$, we have $\operatorname{Tor}_{p}^{K}\left(X_{s}, B\right)=0$ for all $p>0$. Therefore it follows from $[1$, Chapter XV, 7.6a] that

$$
\operatorname{Tor}_{p}^{K(G)}\left(X_{s} \otimes_{K} B, K\right) \approx \operatorname{Tor}_{p}^{K(G)}\left(X_{s}, B\right)
$$

for all $p>0$, where $X_{s} \otimes_{K} B$ is the right $K(G)$-module defined by $(x \otimes b) g=\left(x g \otimes g^{-1} b\right)$ for all $x$ in $X_{s}, b$ in $B, g$ in $G$. Since w. $\operatorname{dim}_{K(G)} K$ $=r$, we have $\operatorname{Tor}_{p}^{K(G)}\left(X_{s} \otimes_{K} B, K\right)=0$ for all $p>r$. Thus it follows from (1) and (2) that $\operatorname{Tor}_{n}^{K(G)}(A, B)=0$ for all $n>s+r$. Therefore w. gl. $\operatorname{dim} K(G) \leqq s+r$.

Proposition 5. Let $G$ be a group and $\left(G_{\alpha}\right)$ a directed family of subgroups of $G$ such that $G$ is the direct limit of the $G_{\alpha}$. If $A$ is a left $K(G)$ module, we have

$$
\text { w. 1. } \operatorname{dim}_{K(G)} A=\sup _{G_{\alpha}} \text { w. 1. } \operatorname{dim}_{K\left(G_{\alpha}\right)} A .
$$

From this it follows that

$$
\text { w. gl. } \operatorname{dim} K(G)=\sup _{G_{\alpha}} \text { w. gl. } \operatorname{dim} K\left(G_{\alpha}\right) .
$$

Proof. Since $G$ is the direct limit of the $G_{\alpha}$, it follows that $K(G)$ is the direct limit of the $K\left(G_{\alpha}\right)$. By [1, Chapter VI, Exercise 17] we have

$$
\operatorname{Tor}_{n}^{K(G)}(C, A)=\lim _{\rightarrow} \operatorname{Tor}_{n}^{K\left(G_{\alpha}\right)}(C, A) \text { (direct limit) }
$$

for all $n$ and all right $K(G)$-modules $C$ and all left $K(G)$-modules $A$. Therefore we see that w. $1 . \operatorname{dim}_{K(G)} A \leqq \sup _{G_{\alpha}}$ w. $1 . \operatorname{dim}_{K\left(G_{\alpha}\right)} A$. On the other hand, we know by Proposition 3 that for each $G_{\alpha}$,

$$
\text { w. l. } \operatorname{dim}_{K(\sigma)} \geqq \text { w. } 1 . \operatorname{dim}_{K\left(\theta_{\alpha}\right)} A \text {. }
$$

This establishes the first equality. A similar argument proves the second equality.

Proposition 6. Let $G$ be a locally finite group. Then the following statements are equivalent:

(a) w. $1 . \operatorname{dim}_{K(G)} K=0$;

(b) w. $1 . \operatorname{dim}_{K(G)} K<\infty$;

(c) $K$ is uniquely divisible by the order of each element in $G$. 
Proof. (a) $\Rightarrow$ (b). Obvious.

(b) $\Rightarrow$ (c). Let $G^{\prime}$ be a cyclic subgroup of $G$ of order $n$. Since the cohomology groups of a group depend only on the group and not on the ground ring $K$ (see $[1, \mathrm{X}, 3.1]$ ), we have by [1, XII, 7] that $\operatorname{Tor}_{2 q}^{K\left(G^{\prime}\right)}(K, K)=K / n K$ (for all $q>0$ ) and $\operatorname{Tor}_{2 q-1}^{K\left(G^{\prime}\right)}(K, K)$ is the subgroup of $K$ consisting of all $k$ such that $n k=0$ (for all $q>0$ ). By Proposition 3, we know that w. $1 . \operatorname{dim}_{K(G)} K \geqq$ w. $1 . \operatorname{dim}_{K\left(G^{\prime}\right)} K$. Therefore $\operatorname{Tor}_{q}^{K\left(G^{\prime}\right)}(K, K)=0$ for all $q>0$, which means that $K$ is uniquely divisible by $n$.

(c) $\Rightarrow\left(\right.$ a). Let $G^{\prime}$ be a finitely generated subgroup of $G$. Then $G^{\prime}$ is a finite group whose order we will denote by $n$. Define the $K\left(G^{\prime}\right)$ homomorphism $f: K \rightarrow K\left(G^{\prime}\right)$ by $f(k)=(k / n) \sum_{o \in G} g$. Then we have $K \rightarrow K\left(G^{\prime}\right) \rightarrow^{\bullet} K$ and $\epsilon f$ is the identity map of $K$ into $K$. Thus $K$ is a direct summand of $K\left(G^{\prime}\right)$ and therefore w. $1 . \operatorname{dim}_{K\left(G^{\prime}\right)} K=0$. Since $G$ is the direct limit of all the finitely generated subgroups $G^{\prime}$ of $G$, we have by Proposition 5 that w. $1 . \operatorname{dim}_{K(G)} K=0$.

Combining Propositions 4 and 6 we have

THEOREM 7. If $G$ is a locally finite group and w. $1 . \operatorname{dim}_{K(G)} K<\infty$, then

w. gl. $\operatorname{dim} K(G)=$ w. gl. $\operatorname{dim} K$.

In particular, $K(G)$ is regular if and only if $K$ is regular and is uniquely divisible by the order of each element in $G$.

Lemma 8. If $G$ is an infinite cyclic group, then w. $\operatorname{dim}_{K(G)} K=1$. Further, if $K$ is a regular ring, then w. $\mathrm{gl} . \operatorname{dim} K(G)=1$.

Proof. Let $\epsilon: K(G) \rightarrow K$ be the unit augmentation. If $g$ is a generator for $G$, it is easy to show that $I(G)=\operatorname{Ker} \epsilon$ is a free left $K(G)$ module with basis $(g-1)$. Thus the sequence

$$
0 \rightarrow K(G) \stackrel{f}{\rightarrow} K(G) \rightarrow K \rightarrow 0
$$

where $f(x)=(1-g) x$, is a projective resolution of $K$. It follows that w. 1. $\operatorname{dim}_{K(G)} K \leqq 1$. Since $\operatorname{Tor}_{1}^{K(G)}(K, K)=\operatorname{Ker}\left(K(G) \otimes_{K(G)} K\right.$ $\left.\rightarrow K(G) \otimes_{K(G)} K\right)=K \neq 0$, we see that w. $1 . \operatorname{dim}_{K(G)} K=1$. The rest of the lemma follows from Proposition 4.

THEOREM 9. If $K(G)$ is a regular ring, then $G$ is a torsion group and $K$ is uniquely divisible by the order of each element in $G$.

Proof. If $G^{\prime}$ is an infinite cyclic subgroup of a group $G$, we have by Proposition 3 and Lemma 7 that w. gl. $\operatorname{dim} K(G) \geqq$ w. gl. $\operatorname{dim} K\left(G^{\prime}\right)$ $\geqq$ w. $1 . \operatorname{dim}_{K\left(G^{\prime}\right)} K=1$. Thus if $K(G)$ is a regular ring, $G$ is a torsion 
group. Since $K(G)$ is regular, we have that w. $1 . \operatorname{dim}_{K(G)} K=0$. Applying Proposition 6, we have the desired conclusion.

Proposition 10. Let $G$ be a finitely generated commutative group and $K$ a Noetherian ring. Then $K(G)$ is a Noetherian ring.

Proof. Trivial.

Proposition 11. Let $G$ be a finitely generated commutative group and $K$ a field. Then we have

$$
\text { w. gl. } \operatorname{dim} K(G)=\text { w. } \lim _{K(G)} K=\operatorname{dim}_{K(G)} K,
$$

where $\operatorname{dim}_{K(G)} K$ stands for the projective dimension of $K$ over $K(G)$.

Proof. The first equality follows from Proposition 4. The second equality follows from the fact that for Noetherian rings the weak dimension of a finitely generated module equals the projective dimension [1, VI, Exer. 3].

Proposition 12. Let $G$ be a finitely generated commutative group whose torsion subgroup has order $n$. If $K$ is a field of characteristic $p$ not dividing $n$, then

$$
\text { w. gl. } \operatorname{dim} K(G)=\operatorname{rank} \text { of } G \text {. }
$$

Proof. By induction on $r=\operatorname{rank}$ of $G$. If $r=0$, then by Theorem 8 , $K(G)$ is regular i.e. w. gl. $\operatorname{dim} K(G)=0$. Suppose $r=k+1 \geqq 1$. Then $G=G^{\prime} \times G^{\prime \prime}$ (direct product) where $G^{\prime \prime}$ is infinite cyclic and $G^{\prime}$ has rank $k$. By $[1, \mathrm{XI}, 3.2]$ we have

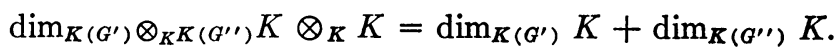

Since $K\left(G^{\prime}\right) \otimes_{K} K\left(G^{\prime \prime}\right)=K(G)$ and $K \otimes_{K} K=K$, we have by Proposition 12 ,

$$
\text { w. gl. } \operatorname{dim} K(G)=\text { w. gl. } \operatorname{dim} K\left(G^{\prime}\right)+\text { w. gl. } \operatorname{dim} K\left(G^{\prime \prime}\right) \text {. }
$$

By Lemma 9 we have w. gl. $\operatorname{dim} K\left(G^{\prime \prime}\right)=1$. Since $G^{\prime}$ has rank $k$ and the torsion subgroup of $G^{\prime}$ is the same as the torsion subgroup of $G$, we have w. gl. $\operatorname{dim} K\left(G^{\prime}\right)=k$. Thus w. gl. $\operatorname{dim}(G)=k+1=\operatorname{rank}$ of $G$.

THEOREM 13. If $G$ is a commutative group and $K$ is a field of characteristic not dividing the order of any torsion element in $G$, then

$$
\text { w. gl. } \operatorname{dim} K(G)=\operatorname{rank} \text { of } G \text {. }
$$

Proof. Since $G$ is the direct limit of its finitely generated subgroups, $G_{\alpha}$, we have by Proposition 5 that w. gl. $\operatorname{dim} K(G)$ $=\sup _{a_{\alpha}}$ w. gl. $\operatorname{dim} K\left(G_{\alpha}\right)$. The theorem now follows from Proposition 13. 


\section{REFERENCES}

1. H. Cartan and S. Eilenberg, Homological algebra, Princeton University Press, 1956.

2. M. Harada, Note on the dimension of modules and algebras, J. Inst. Polytechnics, Osaka City University vol. 7 (1956).

UNIVERSity OF MichigAN

\section{A CHARACTERIZATION OF SOME METACYCLIC GROUPS}

\section{EUGENE SCHENKMAN ${ }^{1}$}

Szasz [1] has recently shown that a group is cyclic if and only if it satisfies condition (A) below.

(A) Every cyclic subgroup of the group is for some positive integer $k$ the subgroup generated by the $k$ th powers of the elements of the group.

We shall extend this idea here to show that a metacyclic group whose commutator subgroup has order relatively prime to its index is characterized as a solvable group satisfying condition (B) below.

(B) Every member of a composition series (i.e. every subinvariant subgroup) is for some positive integer $k$ the subgroup generated by the $k$ th powers of the elements of the group. (If $G$ denotes the group, the subgroup will be denoted by $G(k))$.

If the hypothesis of solvability is not included it is easy to check that many completely reducible groups and their extensions, (including, for example, all simple groups and all symmetric groups) satisfy condition (B). We were unable to characterize these.

We first list some of the properties of a group satisfying condition (B).

(1) Every subinvariant subgroup is a fully invariant subgroup of $G$; that is, it is mapped into itself by all endomorphisms of $G$.

For this is true for all $G(k)$.

(2) Every homomorphic image of a group satisfying (B) itself also satisfies (B).

For the generators of corresponding normal subgroups are $k$ th 1956.

Presented to the Society, February 23, 1957; received by the editors December 10,

1 The author is indebted to Professor L. I. Wade for some of the ideas of the paper; he is indebted to the National Science Foundation for support. 